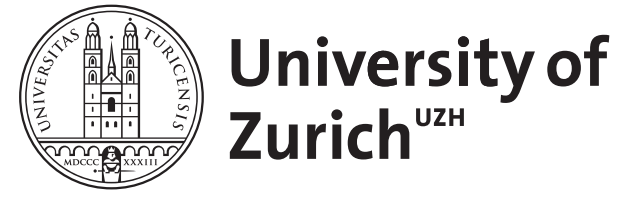

\title{
Spectrally resolved single-molecule electrometry
}

\author{
Ruggeri, Francesca ; Krishnan, Madhavi
}

\begin{abstract}
Escape-time electrometry is a recently developed experimental technique that offers the ability to measure the effective electrical charge of a single biomolecule in solution with sub-elementary charge precision. The approach relies on measuring the average escape-time of a single charged macromolecule or molecular species transiently confined in an electrostatic fluidic trap. Comparing the experiments with the predictions of a mean-field model of molecular electrostatics, we have found that the measured effective charge even reports on molecular conformation, e.g., folded or disordered state, and non-uniform charge distribution in disordered proteins or polyelectrolytes. Here we demonstrate the ability to use the spectral dimension to distinguish minute differences in electrical charge between individual molecules or molecular species in a single simultaneous measurement, under identical experimental conditions. Using one spectral channel for referenced measurement, this kind of photophysical distinguishability essentially eliminates the need for accurate knowledge of key experimental parameters, otherwise obtained through intensive characterization of the experimental setup. As examples, we demonstrate the ability to detect small differences $(5 \%)$ in the length of double-stranded DNA fragments as well as single amino acid exchange in an intrinsically disordered protein, prothymosin .
\end{abstract}

DOI: https://doi.org/10.1063/1.5008936

Posted at the Zurich Open Repository and Archive, University of Zurich ZORA URL: https://doi.org/10.5167/uzh-143926

Journal Article

Published Version

Originally published at:

Ruggeri, Francesca; Krishnan, Madhavi (2018). Spectrally resolved single-molecule electrometry. Journal of Chemical Physics, 148(12):123307.

DOI: https://doi.org/10.1063/1.5008936 


\section{Spectrally resolved single-molecule electrometry}

F. Ruggeri, and M. Krishnan

Citation: The Journal of Chemical Physics 148, 123307 (2018);

View online: https://doi.org/10.1063/1.5008936

View Table of Contents: http://aip.scitation.org/toc/jcp/148/12

Published by the American Institute of Physics

\section{Articles you may be interested in}

Communication: Mechanochemical fluctuation theorem and thermodynamics of self-phoretic motors The Journal of Chemical Physics 147, 211101 (2017); 10.1063/1.5008562

Mixed quantum/semiclassical wave-packet dynamical method for condensed-phase molecular spectroscopy signals

The Journal of Chemical Physics 147, 224112 (2017); 10.1063/1.5003386

A simple model for electrical charge in globular macromolecules and linear polyelectrolytes in solution The Journal of Chemical Physics 146, 205101 (2017); 10.1063/1.4983485

Methods to locate saddle points in complex landscapes

The Journal of Chemical Physics 147, 204104 (2017); 10.1063/1.5012271

Communication: Coordinate-dependent diffusivity from single molecule trajectories

The Journal of Chemical Physics 147, 201102 (2017); 10.1063/1.5006456

Benchmarks of electronically excited states: Basis set effects on CASPT2 results

The Journal of Chemical Physics 133, 174318 (2010); 10.1063/1.3499598

\section{AIP $\left.\right|^{\text {The Journal of }}$ Chemical Physics}

\section{PERSPECTIVES}




\title{
Spectrally resolved single-molecule electrometry
}

\author{
F. Ruggeri ${ }^{1}$ and M. Krishnan ${ }^{1,2, a)}$ \\ ${ }^{1}$ Department of Chemistry, University of Zürich, Winterthurerstrasse 190, CH 8057 Zürich, Switzerland \\ ${ }^{2}$ Department of Physics, University of Zürich, Winterthurerstrasse 190, CH 8057 Zürich, Switzerland
}

(Received 11 October 2017; accepted 20 November 2017; published online 13 December 2017)

\begin{abstract}
Escape-time electrometry is a recently developed experimental technique that offers the ability to measure the effective electrical charge of a single biomolecule in solution with sub-elementary charge precision. The approach relies on measuring the average escape-time of a single charged macromolecule or molecular species transiently confined in an electrostatic fluidic trap. Comparing the experiments with the predictions of a mean-field model of molecular electrostatics, we have found that the measured effective charge even reports on molecular conformation, e.g., folded or disordered state, and non-uniform charge distribution in disordered proteins or polyelectrolytes. Here we demonstrate the ability to use the spectral dimension to distinguish minute differences in electrical charge between individual molecules or molecular species in a single simultaneous measurement, under identical experimental conditions. Using one spectral channel for referenced measurement, this kind of photophysical distinguishability essentially eliminates the need for accurate knowledge of key experimental parameters, otherwise obtained through intensive characterization of the experimental setup. As examples, we demonstrate the ability to detect small differences $(\sim 5 \%)$ in the length of double-stranded DNA fragments as well as single amino acid exchange in an intrinsically disordered protein, prothymosin $\alpha$. Published by AIP Publishing. https://doi.org/10.1063/1.5008936
\end{abstract}

\section{INTRODUCTION}

Electrical charge is a fundamental property of biological matter and plays a central role in the function and interaction of biological molecules. ${ }^{1,2}$ For example, "supercharged" isoforms of evolutionarily conserved proteins are known to confer extreme physiological capacities on certain species, presumably owing to their enhanced stability to aggregation at high concentration. $^{3-5}$ It is also well known that the addition and removal of small amounts of structural charge in the form of phosphate groups or other post-translational modifications modulate not only such basic phenomena as protein stability but also sub-cellular localization or function and can regulate macroscopic processes such as metabolism at the systemic levels. ${ }^{6}$ A recent study demonstrated at the molecular level that the addition of as few as two phosphate groups to a protein induced a folding-unfolding transition and altered its binding affinity by orders of magnitude. ${ }^{7}$ Not surprisingly, several disease states are correlated with altered phosphorylation of proteins, e.g., disordered proteins such as tau and $\alpha$-synuclein involved in Alzheimer's and Parkinson's disease, and multimeric proteins such as the stress-activated p53, implicated both in ageing and cancer. ${ }^{8,9}$ Furthermore there is strong evidence that molecular electrostatics plays a dominant role in phase segregation processes mediated by charged disordered proteins and nucleic acids in the cell. ${ }^{10,11}$ The ability to perform a direct, sensitive, high-resolution measurement of the charge of a macromolecule in solution would thus have strong

a)Electronic mail: madhavi.krishnan@uzh.ch implications from both a fundamental as well as a biomedical perspective.

Contrary to the situation in vacuum, the electrical charge of a macromolecule in solution is governed strongly by thermodynamic processes in the electrolyte that render both theoretical predictions and experimental measurements of the quantity non-trivial. At the simplest level, a direct sum over a macromolecule's charged groups yields a qualitative estimate of its formal structural electrical charge, at a given solution $\mathrm{pH}$,

$$
q_{\mathrm{str}}=\sum_{i} \frac{z_{i} e}{1+10^{z_{i}\left(\mathrm{pH}-\mathrm{p} K_{i}\right)}} .
$$

Here $i$ denotes each ionizable group, $\mathrm{p} K_{i}$ is the negative logarithm of its acid dissociation constant, $z_{i}=+1$ or -1 indicates the valence of charge of a basic or an acidic group, respectively, and $e$ is the elementary charge. In practice however, collective interactions in a densely packed system of charges can dramatically modify the molecule's effective charge in solution via two separate phenomena, namely, charge regulation and charge renormalization. The former concerns an alteration in the charged state of an ionizable group in the context of the molecular environment, while the latter deals with the highly non-linear screening of molecular charge by counterions in the surrounding electrolyte phase. Both phenomena generally result in a reduced "effective" charge of an electrically charged object and have each received extensive theoretical attention, from polyelectrolytes and proteins to colloidal particles and charged surfaces in solution. ${ }^{12-19}$

A variety of experimental approaches have been applied to the problem of electrical charge measurement on a nanoscale entity in the fluid phase. Electrokinetic methods that probe 
the electrical potential ( $\zeta$-potential) near the surface of an object in an applied electric field yield qualitatively correct results, but the measurement is not highly sensitive at the quantitative level. This is due at least in part to the highly non-linear and even non-monotonic response of a particle's electrical mobility to its charge. ${ }^{20}$ Along similar lines, capillary electrophoresis can offer single-charge resolved detection of weakly charged species, but the strong dependence of mobility on an a priori unknown drag coefficient renders direct determination of charge on an unknown molecule difficult; in some instances, chemically generated "charge ladders" of the protein of interest circumvent this issue. ${ }^{21}$ Notwithstanding, polarization fields and molecular deformation in an applied field inherently limit the ability of the electrokinetic approach to sensitively probe the charge of a macromolecule in solution. ${ }^{20}$ Moving out of the solution-phase, native electrosprayionization mass spectrometry can generate high resolution charge spectra of a macromolecule of interest, but there is considerable debate on whether and how the measured spectrum relates to the charge of the molecule in solution, with measurements often contradicting each other even at the qualitative level. ${ }^{22,23}$

We recently demonstrated the ability to measure the effective charge of a single macromolecule in solution with subelementary charge precision and in real time. ${ }^{24}$ The method relied on our recently developed equilibrium thermodynamicsbased approach to trapping electrically charged matter in solution. ${ }^{25}$ Such a trap does not require externally applied fields, but rather exploits the equilibrium repulsive electrostatic interaction between a charged object in solution and like-charged confining parallel plates. Geometric tailoring of the parallel plates results in a local interaction potential minimum that is capable of confining an electrically charged molecule for long periods. ${ }^{25,26}$

For an object confined in a potential well in the fluid phase, the escape process is governed by overdamped diffusive crossing of an energy barrier and is well described by Kramers' theory in the regime $W>6 k_{\mathrm{B}} T .{ }^{24}$ Here the average time to escape the potential well is given by

$$
t_{\mathrm{esc}}=t_{\mathrm{r}} \exp \frac{W}{k_{\mathrm{B}} T},
$$

where $t_{\mathrm{r}}$ is a time scale representing the position relaxation time of the molecule, which in turn depends not only on geometric features of the potential well but also on the molecule's diffusion coefficient, $D=k_{\mathrm{B}} T / 6 \pi \eta r_{\mathrm{H}}$. Here, $r_{\mathrm{H}}$ is the hydrodynamic radius of an equivalent sphere that experiences the same frictional drag as the object of interest, and $\eta$ is the viscosity of the medium. ${ }^{27}$

Relying on this strong non-linear dependence, we converted the measured average escape time of a trapped molecule into a highly precise measurement of the barrier height, $W$. We note that the barrier height in our case is in essence the interaction free energy of the molecule with the confining parallel-plate slit relative to infinite separation. ${ }^{28,29}$ It is also worth noting that this electrostatic interaction energy, $W$, expressed as $W=q_{\mathrm{eff}} \psi_{\mathrm{m}}+f$, directly depends on $q_{\mathrm{eff}}$, the molecule's effective charge. $q_{\text {eff }}$ is in turn not only a function of the molecule's true structural charge but notably also of its
3D conformation..$^{29}$ Here $\psi_{\mathrm{m}}$ is the electrical potential at the midplane of the slit and $f$ is a small contribution to the total free energy from the finite out-of-plane thermal fluctuations of the molecule.

Importantly, the high measurement precision offered by the approach stems from the exponential dependence of the measurand (the escape time, $t_{\mathrm{esc}}$ ) on the measurable (the measured effective charge of the molecule, $q_{\mathrm{m}}$ ), as reflected in Eq. (2). Since escape times are exponentially distributed, the fractional measurement uncertainty on $t_{\text {esc }}$ from a sample of $N$ statistically independent "hops" of a molecule is simply $1 / \sqrt{N}$. Since $q_{\mathrm{m}} \simeq \ln \left(\frac{t_{\mathrm{esc}}}{t_{\mathrm{r}}}\right) \frac{k_{\mathrm{B}} T}{\psi_{\mathrm{m}}}$, the corresponding fractional uncertainty on $q_{\mathrm{m}}$ is given by $\frac{k_{\mathrm{B}} T}{q_{\mathrm{m}} \psi_{\mathrm{m}}} \frac{1}{\sqrt{N}} .{ }^{24}$ Thus for $N=100$, the logarithmic dependence of $q_{\mathrm{m}}$ on $t_{\mathrm{esc}}$ implies $\sim 2 \%$ precision in determining $q_{\mathrm{m}}$ in a measurement time on the order of $10 \mathrm{~s}$. This level of measurement precision on the electrical charge of an entity in solution is unprecedented.

Furthermore, the ability to perform the measurement on a single molecule immediately raises the prospect of detecting tiny differences between individual molecules in a heterogeneous distribution of molecular states arising from, e.g., different phosphorylation patterns, mutations in charged residues, or different conformational states. Thus the Escape-Time Electrometry (ETe) approach offers tremendous scope for detection and measurement of structural, conformational, and compositional heterogeneity of macromolecules in solution, e.g., folding intermediates, mutations in proteins, and posttranslational modifications such as phosphorylation, to name a few.

Although a statistically limited experimental error of say $<5 \%$ on $t_{\text {esc }}$ implies a precision of $<2 \%$ in charge determination in a single measurement, the accuracy in a single measurement is not expected to be as high. This is because in practice, under our experimental conditions, the experimental uncertainty of $\sim 1 \mathrm{~nm}$ on the slit height results in an estimated overall peak-to-peak single-measurement uncertainty of $20 \%$ in charge determination. ${ }^{24}$ In our original demonstration, this device-related measurement uncertainty was reduced to the level of $2 \%-6 \%$ by averaging over several independent measurements. ${ }^{24}$ We did note however that a sequential electrometry measurement on different molecular species in a single device permitted us to address some of the variability in experimental parameters and to thus approach statistically limited precision in a single measurement. Thereby, we demonstrated the ability to distinguish between species carrying very similar amounts of charge. ${ }^{24}$ Nonetheless, given the high precision offered by the method, the prospect of attaining both precision and accuracy in a single measurement on different species or molecules in parallel would be highly desirable.

Here we examine the possibility of a parallel electrometry measurement on spectrally distinguishable molecules under identical experimental conditions in a single experiment. If one of the molecules in the measurement serves the function of a "calibrator," then the charge of the other spectrally distinct species-or single molecule-can be determined with an accuracy approaching the statistically limited precision. Alternatively, if absolute values of effective charge are not 
of interest, but the aim is rather to detect minute differences between molecules, here again the parallel, spectrally distinguishable approach would eliminate all other potential sources of variation and enable the attainment of measurement resolution approaching the statistical limit. We demonstrate this new measurement principle and approach using fluorescently labeled nucleic acid fragments and an intrinsically disordered protein, prothymosin $\alpha(\operatorname{ProT} \alpha){ }^{30,31}$

\section{MATERIALS AND METHODS}

\section{A. Fluorescently labeled biomolecules}

All DNA oligomers in our experiments were purchased from either Microsynth AG (Switzerland) or IBA GmbH (Germany) and carried two fluorescent labels, either ATTO 488 or ATTO 532. The dyes were linked to the $5^{\prime}$ and $3^{\prime}$ termini in the case of ssDNA and to both $5^{\prime}$ termini for the doublestranded fragments. We also examine a $60 \mathrm{bp}$ dsDNA fragment carrying a single ATTO 488 label on the $5^{\prime}$ end of a single strand and a second identical dye an interior location of the complementary strand [Fig. 3(a)]. In the latter case, the dye was linked via an amino dT C6 modification to Thymine 30. A modified version of the intrinsically disordered protein, prothymosin $\alpha(\operatorname{ProT} \alpha),{ }^{30,31}$ carrying a single amino acid exchange E59K was produced using site directed mutagenesis as previously described. ${ }^{24}$ Both ProT $\alpha$ species were labeled with either ATTO 488 or ATTO 532 maleimide (ATTO-Tec, Germany) at cysteine residues.

\section{B. Optical setup and electrometry measurements}

In this work, we use free energy landscapes created in parallel-plate $\mathrm{SiO}_{2}$ slits of typical nominal height, $2 h=70-90 \mathrm{~nm}$, containing surface nanostructured indentations of diameter $600 \mathrm{~nm}$ [Fig. 1(b)] and depth $150 \mathrm{~nm}$, as previously described. ${ }^{24}$ A suspension of fluorescently labeled DNA or protein in a buffer containing $1 \mathrm{mM}$ Tris and $0.5-1.5 \mathrm{mM} \mathrm{NaCl}$ is introduced into the lattice at a concentration of $10-50 \mathrm{pM}$ by capillary flow. ${ }^{24}$ After a few minutes, the flow is arrested and molecular motion in lattices of traps is imaged under purely diffusive conditions by wide-field
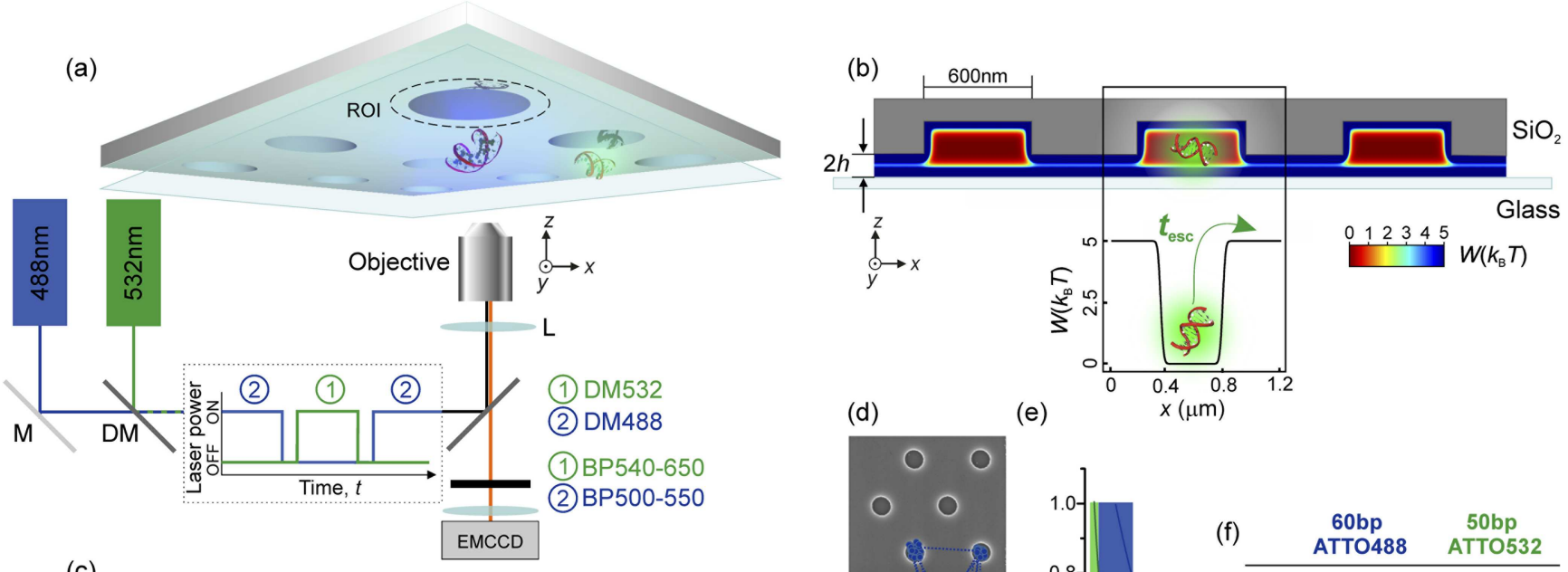

(c)
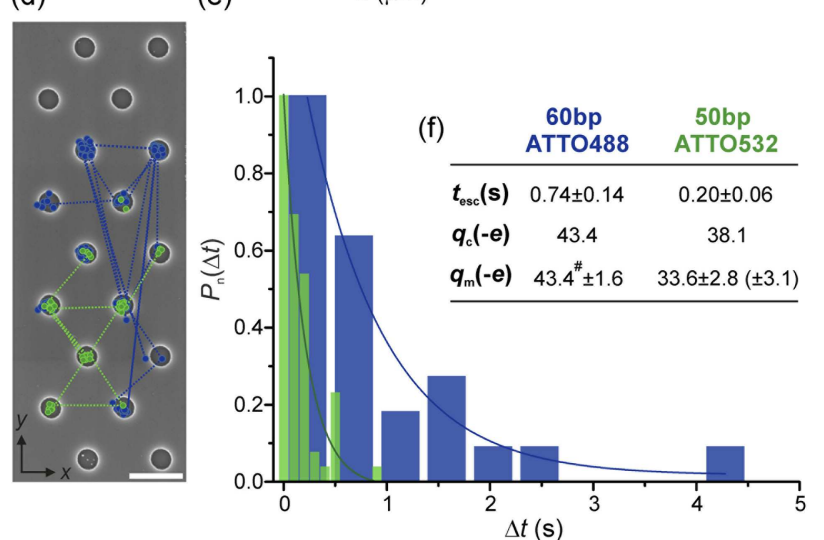

FIG. 1. (a) Schematic representation of the optical setup used for a spectrally resolved single-molecule electrometry experiment. Wide-field fluorescence microscopy employing temporally alternating excitation beams at $488 \mathrm{~nm}$ and $532 \mathrm{~nm}$ is used to image single macromolecules labeled with ATTO 488 and ATTO 532 diffusing in a lattice of electrostatic fluidic traps. M: silver mirror, DM: dichroic mirror, L: lens, BP: bandpass filter, EMCCD: camera. (b) Representative calculation of the free energy landscape, $W(\boldsymbol{r})$ in the lattice of traps. $2 h$ denotes the height of the parallel-plate slit, and the typical nanostructure diameter is $600 \mathrm{~nm}$. (c) The fluorescence intensity of a Region of Interest (ROI) given by the dashed contour in (a), centered on a single trap, monitored in time in both emission channels, clearly shows longer-lived trapped states for 60bp dsDNA-ATTO488 (blue) and shorter trapping times for 50bp dsDNA-ATTO532 (green) (see the supplementary material). (d) Single-particle tracking trajectories of a representative single molecule of each species superimposed on a scanning electron micrograph (SEM) of the underlying nanostructured surface. Scale bar is $1 \mu \mathrm{m}$. (e) Probability density distributions, $P(\Delta t)=\frac{A}{t} \exp (-\Delta t / t)$, of $\operatorname{experimentally}$ recorded escape times $\Delta t$, where $A \approx 1$. The distributions are normalized such that $P_{\mathrm{n}}(\Delta t)=\frac{P(\Delta t)}{P_{\max }(\Delta t)}$. (f) Measured average escape time, $t_{\mathrm{esc}}$, in a single lattice over 2-3 molecules for each species $(N \sim 30)$, and calculated and measured values of effective charge, $q_{\mathrm{c}}$ and $q_{\mathrm{m}}$, respectively, for $60 \mathrm{bp}-\mathrm{ATTO} 488$ and 50bp-ATTO532. ${ }^{\#}$ denotes that $q_{\mathrm{m}}$ for 60bp DNA has been set equal to $q_{\mathrm{c}}$, permitting a direct calibrated measurement for the other species (50bp DNA). Measurement uncertainties are standard error of the mean (s.e.m.). Values indicated in parentheses for 50bp DNA include the measurement uncertainty on the 60bp DNA "calibration molecule." 
fluorescence microscopy. We typically work with landscapes created by a hexagonal lattice of approximately 40 traps where the extent of each lattice is typically $15 \mu \mathrm{m} \times 15 \mu \mathrm{m}$. In this work, we study molecules in 3-10 lattices for each measurement.

We use optical excitation at $488 \mathrm{~nm}$ from a diode laser (PhotonTec Berlin, Germany) and at $532 \mathrm{~nm}$ from a diodepumped solid-state (DPSS) laser (Pusch OptoTech GmbH, Germany). We collect fluorescence emission in two spectral bands-500-550 $\mathrm{nm}$ and 540-650 nm-using two temporally alternating dichroic mirrors. The excitation wavelength alternates synchronously with the corresponding emission filters [Fig. 1(a)]. Images of molecular diffusion in the lattice are acquired using a back-illuminated electron multiplying charge coupled device (EMCCD) camera (iXon, Andor, Inc., United Kingdom) with exposures of duration $t_{\exp }=5-20 \mathrm{~ms}$ and sampling time of 20-100 ms during a total observation time of about $10 \mathrm{~s}$ per molecular trajectory. The depth of the wells, $W$ in this work, is typically $5 k_{\mathrm{B}} T$, yielding trap residence times of $\sim 200 \mathrm{~ms}$. Furthermore, in this work, the Debye length $\kappa^{-1} \approx 0.3 / \sqrt{C}=10 \mathrm{~nm}$ and is the characteristic length scale of screening of electric fields in an electrolyte containing monovalent salt at a concentration $C=10^{-3} \mathrm{~mol} / \mathrm{L}$.

\section{RESULTS}

\section{A. Measurements on the same molecular species labeled with different fluorescent dyes}

A key goal of our electrometry approach is to be able to routinely attain close to $1 \%$ measurement precision, and corresponding resolution, in the measured effective charge on two different molecular species, or even two individual molecules of each species, in a single measurement. Spectral splitting of the signal from the species under consideration seems to offer a promising route to this end, as it offers simultaneous measurement under virtually identical experimental conditions-mainly, slit height, salt concentration, and $\mathrm{p} H$.

In an electrometry measurement using two spectrally distinct labels however, a central interest concerns the contributions of the dye molecules themselves to the total molecular charge. Most water-soluble fluorescent dyes used to label biomolecules carry some amount of net charge (typically 1-2e), either positive or negative. In previous work, we presented measurements of the effective charge of $\operatorname{ProT} \alpha$, labeled with spectrally comparable fluorescent dyes carrying different net charge. ${ }^{24}$ Our measurements did in fact reveal net charge values for molecules labeled with Cy3B and Alexa 546 that were different from those labeled with ATTO 532, showing that the measurement is sensitive to the charge state of the label. An ideal spectrally referenced measurement would involve the use of spectrally distinguishable dye species that alter the charge of the molecule of interest in a quantitatively identical fashion, if they do so at all. In this work, we focus on two fluorescent dye labels ATTO 488 and ATTO 532 whose structures are shown in Fig. 2(a). We chose ATTO 488 and ATTO 532 as they are chemically very similar and are both expected to carry a net charge of $-1 e$, over a wide $\mathrm{pH}$ range. They also offer excellent brightness and the required photostability for our measurement. ${ }^{32,33}$

In order to examine the contribution of spectrally distinct dye molecules to the measurement, we performed parallel ETe measurements as described in Sec. II. We find that the variation in slit height, $2 h$, from one lattice of traps to the next is reflected in the measured time scales and corresponding effective charges of both species are measured in parallel. To further motivate this point, we present dual color ETe data in experiments where the slits are etched by two different methods: a solution phase wet-etch process using Hydrofluoric (HF) acid and a gas-phase reactive-ion etch (RIE). The former is known to result in large variations of slit height from one lattice of traps to the next $\left(h_{e} \sim 2-3 \mathrm{~nm}\right)$, while RIEour method of choice, in general-yields uniform slit heights, albeit with an expected nominal rms uncertainty, $h_{e}$, of around $1 \mathrm{~nm}$.

First, we studied two species of 10 base ssDNA molecules, each labeled at both ends with either ATTO 488 or ATTO 532 in slits fabricated using RIE. We find that parallel spectrally resolved electrometry on both species agrees within the statistically expected precision [Fig. 2(b)] and the variations in the measured average escape time from one lattice to the next are mainly statistically limited, as expected. We find that the uncalibrated values of measured charge for both species, $q_{\mathrm{m}}^{*}$, inferred from averaging over $N \sim 200$ escape events recorded in each spectral channel over all lattices, reveal no measurable difference in charge between the two species $(<0.2 e)$, in keeping with the statistical expectation. This suggests that the two dye species affect the total electrical charge of the molecule in an identical fashion [Fig. 2(c)].

We then studied two species of 60bp dsDNA molecules, each labeled at both $5^{\prime}$ ends with either ATTO 488 or ATTO 532, in a device where the slits were fabricated using etching in HF. Here large correlated variations in the measured escape time scale from one lattice to the next for both measured species reflect common measurement conditions, most likely the local slit height, $2 h$ [Figs. 2(d) and 2(e)]. This results in a broad distribution of inferred uncalibrated charge values, $q_{\mathrm{m}}^{*}$ [Fig. 2(e)]. Using the effective charge of 60bp dsDNA labeled with ATTO 532, known both from a calculation as well as measurements under the same experimental conditions in previous work, we infer the "true" local slit height in each lattice. This permits a calibrated measurement of the charge of the blue-labeled species $\left(q_{\mathrm{m}}\right)$. Figure 2(e) displays both the uncalibrated and spectrally calibrated measured values for the ATTO 488 labeled species. Not only is the mean calibrated value significantly different from the direct measurement but importantly the distribution of measured values is also significantly narrower. These observations exemplify how spectral referencing eliminates a significant source of uncertainty in a single measurement and ultimately enables both accurate and precise measurements on a single molecule [Fig. 2(f)]. We note however that these ensemble-averaged measurements reveal a slightly lower measured charge for the 60bp-ATTO 488 species as compared to the ATTO 532 labeled counterpart. This may be due to either residual measurement noise from the large slit height variation that has not averaged out or more likely the presence of a few weakly charged molecules (possibly a small 

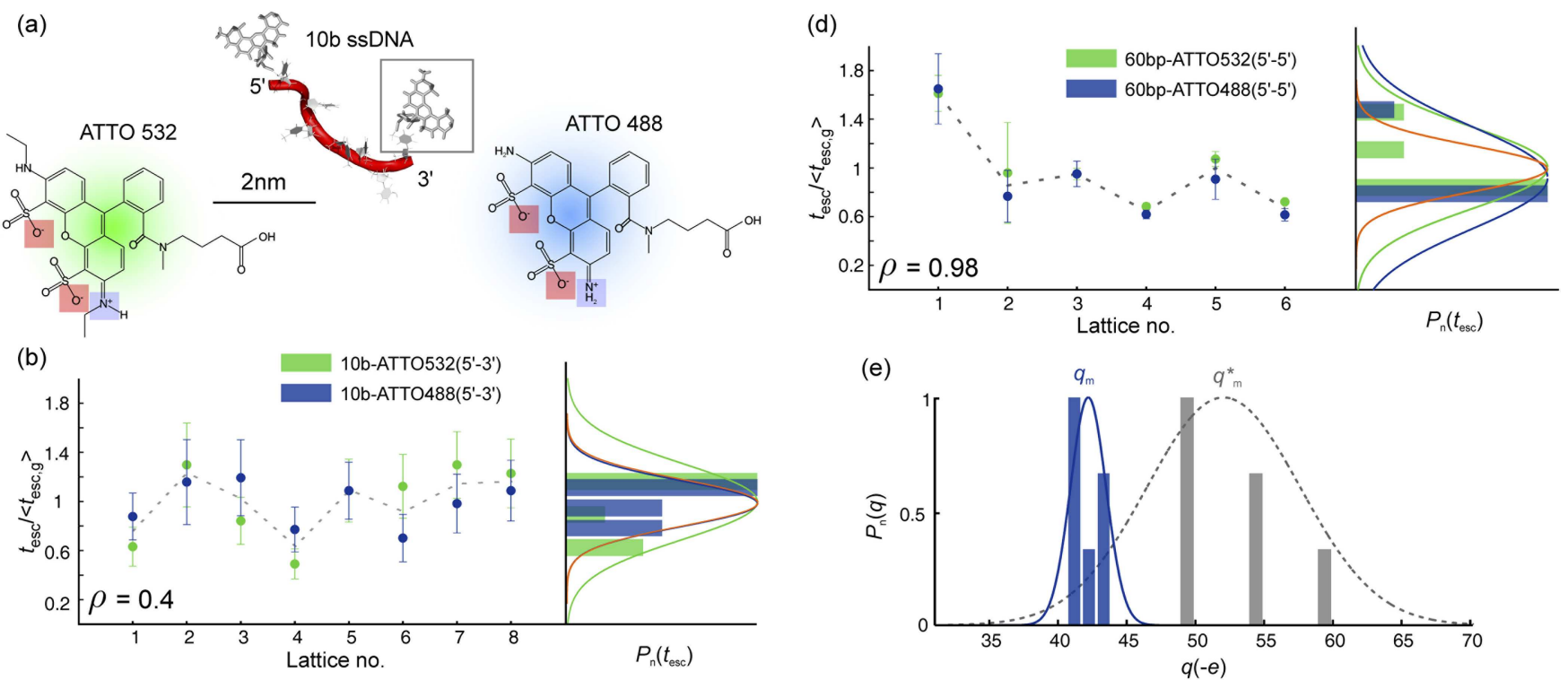

\begin{tabular}{cccc} 
Molecule & $\boldsymbol{t}_{\text {esc }}(\mathrm{ms})$ & $\boldsymbol{q}_{\mathrm{c}}(-\mathrm{e})$ & $\boldsymbol{q}_{\mathrm{m}}^{\boldsymbol{*}}(-\mathrm{e})$ \\
\hline 10b-ATTO532 & $46.7 \pm 5$ & 10.9 & $9.6 \pm 0.5$ \\
10b-ATTO488 & $47.5 \pm 3$ & 10.9 & $9.7 \pm 0.3$ \\
\hline
\end{tabular}

(f) \begin{tabular}{cccc} 
Molecule & $\boldsymbol{q}_{\mathrm{c}}(-\mathrm{e})$ & $\boldsymbol{q}_{\mathrm{m}}^{*}(-\mathrm{e})$ & $\boldsymbol{q}_{\mathrm{m}}(-\mathrm{e})$ \\
\hline 60bp-ATTO532 & 43.4 & $53.6 \pm 1.9$ & $43.4^{\#} \pm 0.5$ \\
60bp-ATTO488 & 43.4 & $52.0 \pm 2.3$ & $42.1 \pm 0.5( \pm 0.7)$
\end{tabular}

FIG. 2. (a) Schematic representations of end-labeled nucleic acid molecules, and the chemical structures of the dyes ATTO 488 and ATTO 532, indicating the expected charged state of the ionizable groups under our measurement conditions (light blue and light red). (b) Values of $t_{\text {esc }}$ for 10 base ssDNA labeled with two different dyes, measured in several lattices of traps in the same experiment, normalized by the average measured value of the ATTO 532 labeled species, $\left\langle t_{\text {esc,g }}\right\rangle$ (green). The dashed line indicates the average $t_{\text {esc }}$ for both species in each lattice. Indicated in parentheses are the locations of the ATTO labels for each molecular species. Histograms of $t_{\mathrm{esc}}$ for each species (right panel) show nearly statistically limited measurement precision (orange curve) in both cases. The blue and green gaussian curves indicate average and standard deviations for each species. (c) The table displays measured $t_{\text {esc }}$ for 10 base ssDNA labeled with either ATTO 488 or 532, averaged over the measurements on 8 lattices $(N \sim 200)$ shown in panel (b). Also indicated are the calculated and measured (uncalibrated) effective charge values, $q_{\mathrm{c}}$ and $q_{\mathrm{m}}^{*}$, respectively. The values of $q_{\mathrm{m}}^{*}$ are in remarkable mutual agreement, suggesting that the two dyes most likely affect the overall effective charge of the molecule in a quantitatively identical fashion. Measurement uncertainties are s.e.m. (d) Values of $t_{\mathrm{esc}}$ for 60bp dsDNA labeled with two different dyes measured in several independent lattices of traps in slits fabricated using HF etching, normalized by the average value of the ATTO 532 labeled species (green). Histograms of $t_{\mathrm{esc}}$ for each species (right) reveal a measurement uncertainty that is larger than the expected statistically limited precision (orange) in both cases. However the mean values for each species averaged over all lattices in both (b) and (d) are in good agreement. While the cross-relation coefficient, $\rho$, is high in (d) indicating strongly correlated variation in measurement due to the variation in slit height, $\rho$ is lower in (b) where the lattice-to-lattice measurement variation is mostly statistically limited. (e) Uncalibrated charge measurements, $q_{\mathrm{m}}^{*}$, on $60 \mathrm{bp}$-ATTO488, inferred from about 5 to 10 molecules per lattice, using the nominal measured slit height, $2 h$ (gray histogram). Calibrated measured values, $q_{\mathrm{m}}$, obtained by fixing the charge of the simultaneously measured 60bp-ATTO532 to the calculated value, $q_{c}=-43.4 e$ (blue histogram). The distribution of measured $q_{\mathrm{m}}$ (blue gaussian curve) is much narrower that $q_{\mathrm{m}}^{*}$ (dashed gray curve). (f) The table presents uncalibrated directly measured effective charge values, $q_{\mathrm{m}}^{*}$ in 6 lattices, averaged over 5-10 molecules per lattice, and the corresponding values obtained upon calibration, $q_{\mathrm{m}}$. Measurement uncertainties are s.e.m.

fraction of unannealed ssDNA) in the 60bp-ATTO488 sample, as will be discussed later.

\section{B. Exploring the effect of dye position: Probing local molecular electrostatics}

For a hypothetical molecule carrying identical ionizable groups on its surface, interaction among the solvent-exposed groups leads to a local non-zero surface electrical potential, $\psi_{\mathrm{s}}$. Since the chemical potential of the protons is constant throughout the system, the non-zero potential in the vicinity of the groups results in a local $\mathrm{pH}$, different from that in the bulk. In fact the greater the magnitude of electrical potential at the charged groups, the stronger the departure of their degree of ionization, $\alpha$, from that in isolation, as given by Eq. (3), ${ }^{16,34}$

$$
\alpha=\frac{1}{1+10^{z_{i}(\mathrm{pH}-\mathrm{p} K)} \exp \left(z_{i} e \psi_{\mathrm{s}} / k_{\mathrm{B}} T\right)} .
$$

This behavior essentially embodies the phenomenon termed "charge regulation." Equation (3) can be generalized to any local potential, $\psi$, and can therefore be used to describe the behavior of more complex charge distributions and local environments, including a low-dielectric, non-solvent-exposed milieu representing the interior of folded molecules. ${ }^{29}$

Previous studies have discussed the effect of "supramolecular $\mathrm{p} K_{\mathrm{a}}$ shift" according to which the photophysics and/or charged state of organic dye molecules is altered by the dielectric environment of neighbouring molecules in the host matrix or the local electrostatic environment due to molecule to which the dyes are chemically conjugated. In fact studies on the fluorescein dye have shown that conjugation to ssDNA and dsDNA molecules shifts the dye $\mathrm{p} K_{\mathrm{a}}$ up by $\sim 0.5$ as well as alters the charged state of the fluorophore. ${ }^{35}$ In fluorescein, the phenolic group $\left(\mathrm{p} K_{\mathrm{a}}=6.5\right)$ dissociates to form a dianion which is responsible for intense fluorescence at neutral $\mathrm{pH}$ under excitation at $490 \mathrm{~nm}$. Conjugation to a strongly negatively charged polyelectrolyte molecule such as DNA creates 
a lower $\mathrm{pH}$ in the local environment of the dye moiety as compared to the bulk, which shifts its $\mathrm{p} K_{\mathrm{a}}$ to an apparently higher value, affecting the dye's photophysics. The $\mathrm{p} K_{\mathrm{a}}$ shift of conjugated fluorescein is thus taken to be indicative of the local environment and has even been used to spatially map the local electrostatic potential in RNA molecules. ${ }^{36,37}$

Assuming the linker introduces approximately $1 \mathrm{~nm}$ distance between the nucleic acid and the dye, the electrostatic potential at the end-labeled dye molecule under our measurement conditions is $\psi \sim-75 \mathrm{mV}\left(3 k_{\mathrm{B}} T / e\right)$ [Fig. 3(a)]. Since the dyes are highly acidic $\left(\mathrm{p} K_{\mathrm{a}} \sim 3\right)$ and the measurement $\mathrm{pH}=8.8$, Eq. (3) suggests that the charged state of each endcoupled ATTO dye molecule is not different from its value in free solution. Therefore each dye molecule is expected to add a net structural charge of $-1 e$ to the derivatized molecule
[Fig. 2(a)]. However the renormalized charge contribution of each dye attached to the molecule could be as low as around $-0.5 e$. Indeed our measurements on end-labeled molecules have convincingly demonstrated that the two labels ATTO 488 and ATTO 532 affect the total charge of the molecule in an identical fashion and thus probably have the same ionization behavior. We further address the possibility that local electrostatic effects at the interior of the macromolecule may alter the protonation/deprotonation equilibria of the dye molecule and therefore its charge. If this indeed were the case, the measurement would have to potentially contend with a spurious label-placement dependent contribution to the measured total charge of the molecule.

Figure 3(a) displays the local electrostatic environment of a dye molecule conjugated to an end and an interior location (a)
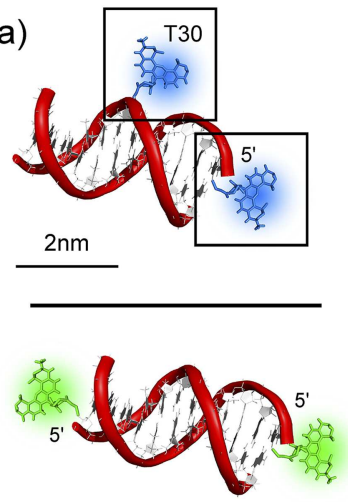
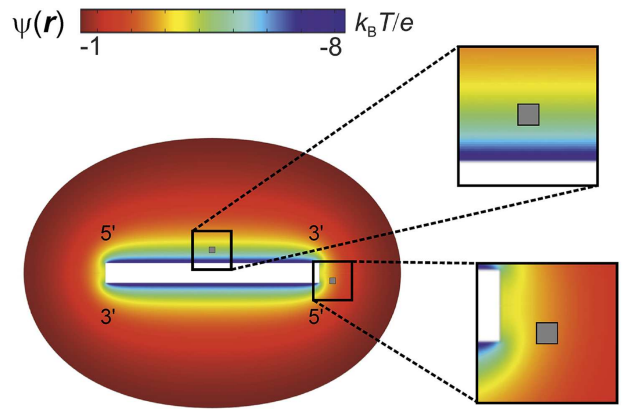

(b)

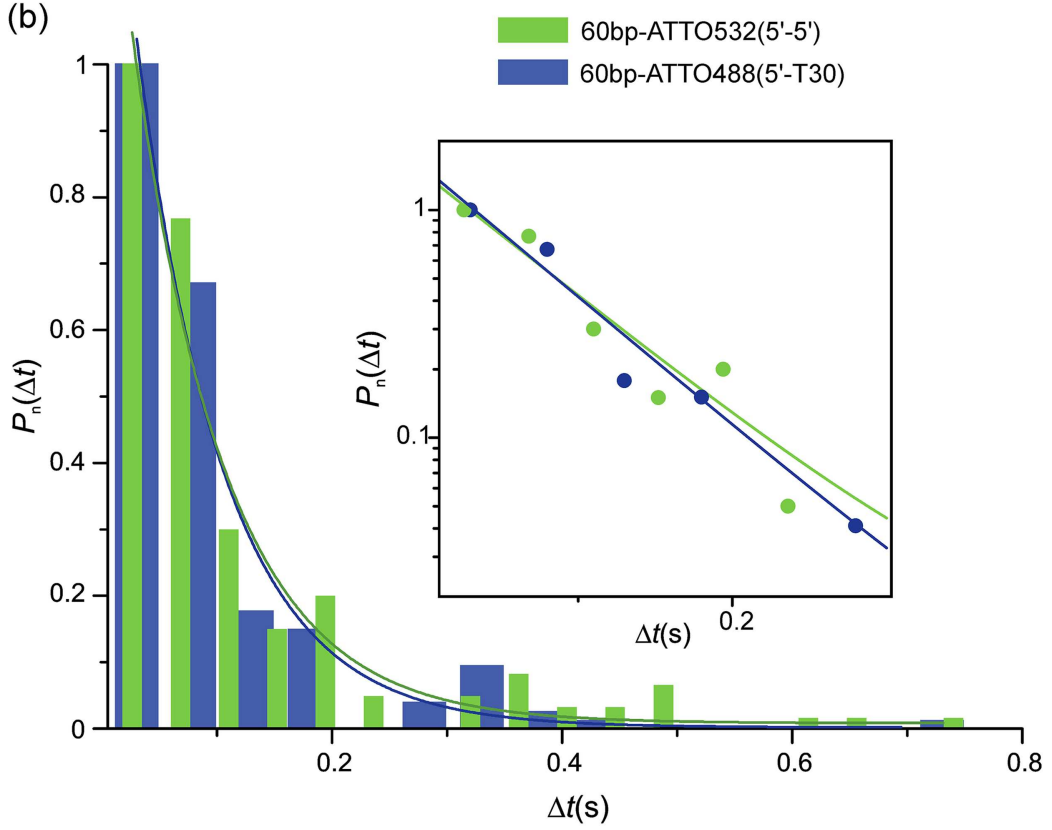

FIG. 3. (a) Schematic representation of two species of 60bp dsDNA molecules, one labeled with ATTO 532 at both $5^{\prime}$ ends and the other labeled with ATTO 488 at the $5^{\prime}$ end and an internal location (T-30). Calculated electrostatic potential distribution, $\psi(\boldsymbol{r})$, around a 60bp dsDNA molecule under our experimental conditions. Gray squares indicate expected approximate locations of the dye molecule relative to the dsDNA fragment, assuming the length of the linker attaching the dye to the molecule is $\sim 1 \mathrm{~nm}$. (b) Normalized probability distributions, $P_{\mathrm{n}}(\Delta t)$, of measured trap times, $\Delta t$, for the two species. (c) The measurements agree within the statistical uncertainty suggesting that dye location has no influence on the measured effective charge. Measurement uncertainties are s.e.m.

(c)

\begin{tabular}{cccc} 
Molecule & $\boldsymbol{t}_{\text {esc }}(\mathbf{s})$ & $\boldsymbol{q}_{\mathrm{c}}(-\mathbf{e})$ & $\boldsymbol{q}_{\mathrm{m}}(-\mathbf{e})$ \\
\hline 60bp-ATTO532 (5'-5') & $0.080 \pm 0.007$ & 43.4 & $43.4^{\#} \pm 1$ \\
60bp-ATTO488 (5'-T30) & $0.076 \pm 0.007$ & 43.4 & $42.8 \pm 1.1( \pm 1.5)$ \\
\hline
\end{tabular}


of a dsDNA fragment. The calculation suggests a difference in electrical potential of around $100 \mathrm{mV}$ between the bulk solution and the interior site of dye attachment. Although these values of local electrical potential (75 and $100 \mathrm{mV}$ ) imply $\mathrm{p} K_{\mathrm{a}}$ shifts of 1.3 units and 1.7 units, respectively, the effect on the charged state of the ATTO dyes is not expected to be significant, especially at our relatively high measurement $\mathrm{p} H \sim 8$.

In order to experimentally probe the influence of local electrostatics on the charged state of the dye label, we compared the measured effective charge of two species of doublelabeled dsDNA molecules. One species of dsDNA carries a single dye label at each end of the molecule, while the other carries a single label at one end and a second label at an interior location. If the comparatively large negative local electrical potential at the interior site were to influence the ionization equilibria of the dye molecule strongly enough to alter its net charge, this would be an important consideration in an electrometry measurement that seeks to measure differences of $\sim 1 e$ between molecules or molecular species.
Our spectrally split parallel electrometry measurement on the two molecular species, which differ only in the location of a single, otherwise effectively identical dye molecule [Fig. 3(a)], reveals no measurable difference in effective charge [Figs. 3(b) and 3(c)]. The reported effective charges of both species of $60 \mathrm{bp}$ dsDNA molecules are based on measurements where the number of hops $N=160$. We expect $3 \%$ measurement resolution under these conditions, which places the expected measurable charge difference between the two spectrally distinguishable species at $\sim 1.3 e$.

We further point out that the midplane electrical potential, $\psi_{\mathrm{m}}$, in this experiment was small by design, resulting in a smaller value of $t_{\text {esc }}$ for $60 \mathrm{bp}$ dsDNA compared to the measurements in Figs. 1(e), 1(f), and 2(d)-2(f). Under these conditions, any spurious weakly charged species in solution (as discussed later) would be very unlikely to contribute a detectable trapped state to the ensemble measurement. Our observations thus strongly suggest that the location of the label does not measurably influence the total effective charge of the molecule. This confirms that the charge of both ATTO 488 and ATTO 532 is unaffected by the highly

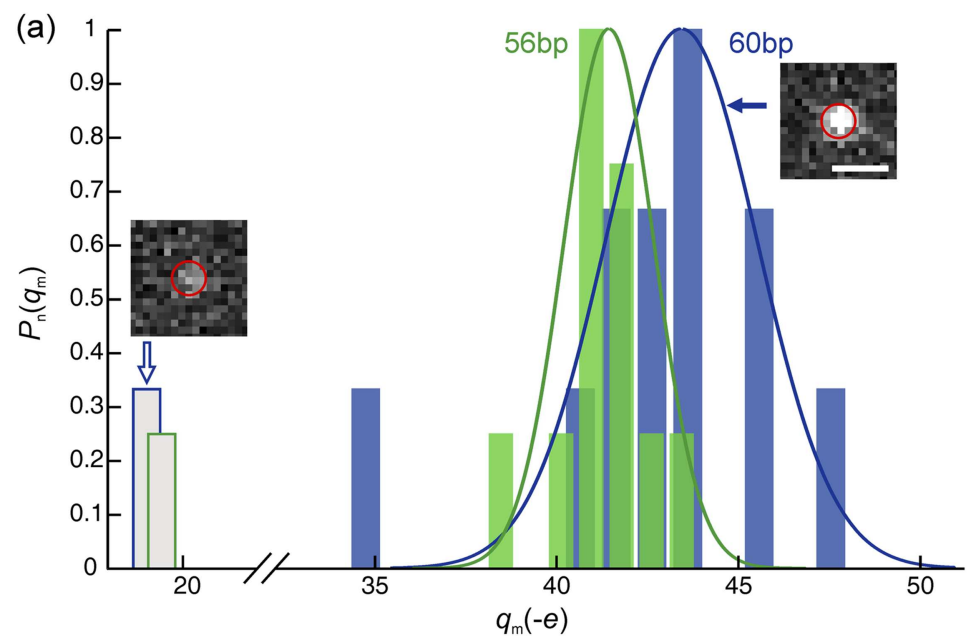

(b)
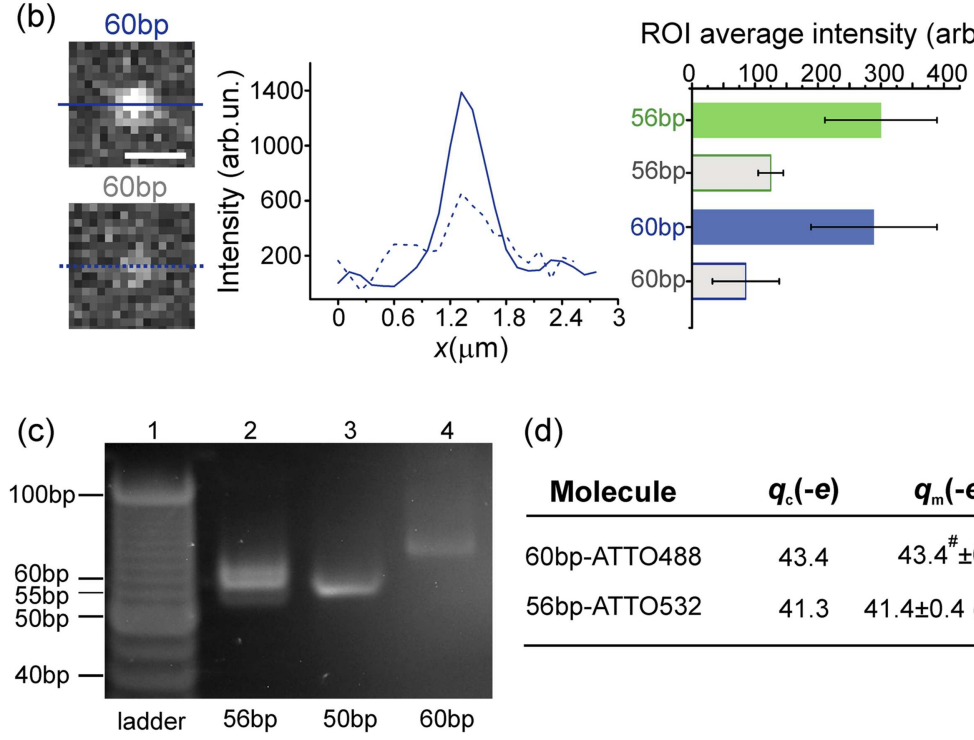

(d)

\begin{tabular}{ccc} 
Molecule & $\boldsymbol{q}_{\mathrm{c}}(-\mathrm{e})$ & $\boldsymbol{q}_{\mathrm{m}}(-\mathrm{e})$ \\
\hline 60bp-ATTO488 & 43.4 & $43.4^{\#}{ } 0.7$ \\
$56 \mathrm{bp}-$ ATTO532 & 41.3 & $41.4 \pm 0.4( \pm 0.8)$ \\
\hline
\end{tabular}

FIG. 4. (a) Histograms of spectrally resolved singlemolecule measurements on 56bp-ATTO532 and 60bpATTO488. Measurements are reported for individual molecules with $N \sim 20$ hops per molecule, resulting in a typical uncertainty of $\sim 2 e$ per measurement on an individual molecule. Solid lines depict gaussian distributions of mean and standard deviation corresponding to the measured dominant fraction of each sample. We also detect the presence of a low abundance weakly charged and weakly emitting species (gray bars). Typical images for molecules in the two fractions are presented in each case for 60bp-ATTO488. Red circles denote the occupied trap. Scale bar is $1 \mu \mathrm{m}$. (b) Typical intensity profiles for the stably trapped, bright and highly charged species (solid line) and the weakly trapped, poorly emitting, low abundance species (dashed line). Bars represent average ROI intensities over all frames where the molecules register as trapped. The combination of weak emission and shortlived trapping suggests that the low abundance species represents a small excess of unannealed single-labeled ssDNA fragments in solution. (c) $20 \%$ native polyacrylamide gel electrophoresis of 5bp DNA ladder (lane 1), 56bp-ATTO532 (lane 2), 50bp-ATTO532 (lane 3), and 60bp-ATTO488 (lane 4) reveal the presence of a rapidly migrating species of low abundance in the $56 \mathrm{bp}$ sample. Molecules in lanes 2-4 display lower mobility than the corresponding fragments in lane 1, likely due to the presence of the dye labels. (d) $q_{\mathrm{m}}$ values of both species, obtained by setting the charge of the dominant fraction of $60 \mathrm{bp}-\mathrm{ATTO} 488$ to $q_{\mathrm{c}}=-43.4 e$. The measurements reveal a difference of about $2 e$ between the species, in excellent agreement with the theoretical expectation. Reported errors are s.e.m. over 11 single-molecule measurements per species. 
negative local electrical potential due to the strongly charged macromolecule.

The insensitivity of these ATTO dyes to local electrostatics is likely the result of their highly acidic sulfonate groups ( $\mathrm{p} K_{\mathrm{a}}<<\mathrm{pH}$, generally). This key feature in combination with their excellent brightness and photostability renders these ATTO dyes excellent labels for spectrally referenced fluorescence-based electrometry measurements.

\section{Measuring small differences of charge in biomolecules}

Finally we explore the prospect of using spectrally resolved single-molecule electrometry in order to detect small differences of charge $(\sim 5 \%)$ in biomolecules such as nucleic acids and proteins.

Parallel electrometry measurements on 50bp dsDNAATTO532 and 60bp dsDNA-ATTO488 reveal a factor 4 difference in $t_{\text {esc }}$ for a modest difference in effective charge of $\sim 12 \%$ [Figs. 1(e) and 1(f)]. This suggests that a measurement uncertainty on $t_{\mathrm{esc}}$ of $\sim 10 \%$, corresponding to $N=100$ hops, should place a charge resolution of $\sim 5 \%$ well within reach.

We therefore performed parallel ETe on 56bp dsDNAATTO532 and 60bp dsDNA-ATTO488, which are expected to differ in effective charge by $2 e$. The histogram in Fig. 4(a) displays the results of $\sim 10$ single molecule measurements that on average yield a difference in electrical charge close to the theoretically expected value. Note that in these measurements, we fix the charge of one species to the theoretical value. As before, this permits us to minimize the uncertainty on the remaining system parameters, thereby enabling both accurate and precise measurements on the unknown molecule.
The data in Fig. 4(a) show that while the two samples are dominated by a species with a long-lived trapped state, the measurement also detects a second, low-abundance species with very rapid escape dynamics. This "fast fraction" also has a much lower optical intensity than the dominant fraction and we find that its effective charge is very close to our previous measurements on doubly labeled 60 base ssDNA $\left(q_{\mathrm{m}}=-26.7 \pm 1.9 e\right)$. Thus we believe that the species represents a sparse population of singly labeled unannealed ssDNA also present in solution. Gel electrophoresis data indeed confirm the presence of a closely migrating species of slightly higher mobility in the 56bp dsDNA sample [Fig. 4(c)].

Interestingly, examining the measurements on isolated molecules - as opposed to an analysis of pooled singlemolecule signals - is key not only to being able to detect small differences between species in different channels but also ultimately to distinguishing different molecular species or states in a given spectral channel.

It is worthwhile to note here that the spatial dimension in the experiment enters the measurement in a subtle way, permitting a chain of nearest neighbor hops in the lattice to be assigned to a distinct molecule. Pooling the measured $\Delta t$ on individual single molecules and extracting an average value for the measurand would wash out small differences between similar species and make it hard, if not impossible, to glean the underlying charge distribution from the escape time measurement. Indeed an ensemble-level measurement of this kind on the two species reveals statistically indistinguishable time scales, $t_{\mathrm{esc}, 60 \mathrm{bp}}=530 \pm 20 \mathrm{~ms}$ and $t_{\mathrm{esc}, 56 \mathrm{bp}}=550 \pm 15 \mathrm{~ms}$. Thus we report a preliminary realization of "charge spectrometry" on an ensemble of single molecules in solution.

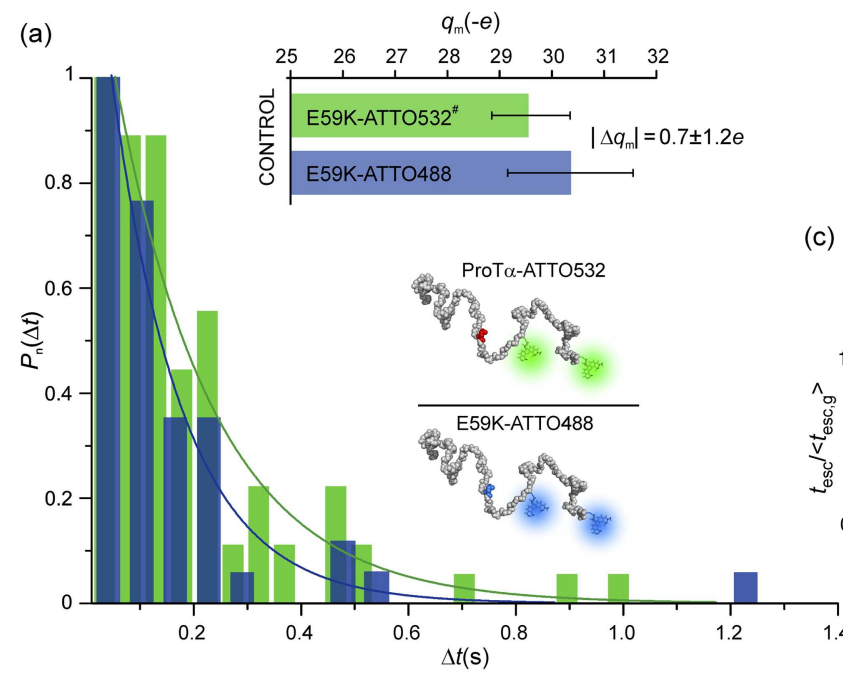

(b)

\begin{tabular}{cccc} 
Molecule & $\boldsymbol{t}_{\text {esc }}(\mathbf{s})$ & $\boldsymbol{q}_{\mathrm{c}}(-\mathrm{e})$ & $\boldsymbol{q}_{\mathrm{m}}(-\mathrm{e})$ \\
\hline ProT $\alpha-$ ATTO532 & $0.180 \pm 0.020$ & 31 & $31^{\#} \pm 1.3$ \\
E59K-ATTO488 & $0.130 \pm 0.014$ & 29.6 & $28.4 \pm 0.8( \pm 1.4)$ \\
\hline
\end{tabular}

(c)

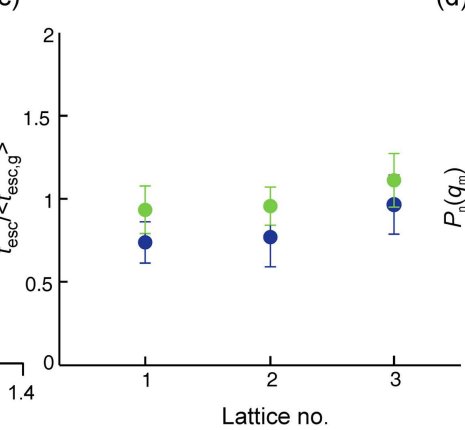

(d)

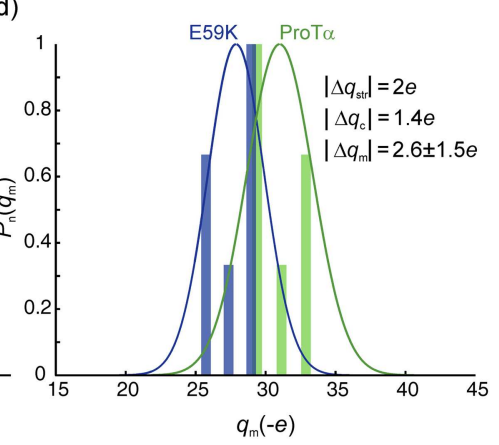

FIG. 5. (a) Histograms of escape times measured for two species of the disordered protein ProT $\alpha$, where the ATTO 488 labeled species carries a single amino acid exchange denoted by E59K [residue highlighted in red (E) and blue (K)]. The histograms represent $N \simeq 60$ events for each species. The blue labeled species clearly reveals a value of $t_{\mathrm{esc}}$ measurably smaller than that of its unmodified counterpart. Control measurements of ProT $\alpha$-E59K, labeled with either ATTO 488 or ATTO 532, reveal no measurable difference in molecular effective charge (top panel). (b) Measured $t_{\text {esc }}$ from (a), converted to $q_{\mathrm{m}}$, setting $q_{\mathrm{m}, \text { ATTO532 }}^{*}=q_{\mathrm{c}}=-31 e$, reveal good agreement with theoretical expectations. Measurement uncertainties are s.e.m. (c) Values of $t_{\mathrm{esc}}$ for each species measured in three lattices of traps in the same experiment, normalized by the average value of the ATTO 532 labeled species (green). ProT $\alpha$ mutant E59K displays a systematically lower $t_{\mathrm{esc}}$ even at the level of the single lattice. (d) Histograms of measured $q_{\mathrm{m}}$ values over 6 lattices, sampled by 2-3 molecules each. Gaussian curves indicate mean and standard deviation for each dataset. 
Finally, we demonstrate the detection of single amino acid exchange in the acidic intrinsically disordered protein $\operatorname{ProT} \alpha$. ProT $\alpha$ is a disordered polypeptide 128 amino acids in length, with a nominal net structural charge of $-46 e$. Most of this charge is contained within a C-terminal stretch of 31 amino acids. Previously we have performed sequential electrometry measurements of ProT $\alpha$ and a mutant version, E59K, which contains a single Glutamate(E) residue replaced by a Lysine $(\mathrm{K}) .{ }^{24}$ Both species were labeled with the same fluorescent dye, ATTO 532, and the measurement revealed a clear separation of escape time scales with a ratio of a factor 2, corresponding to a difference in charge, $\Delta q_{\mathrm{m}}=3 e$, between the species.

We performed spectrally referenced measurements on ATTO 488-labeled ProT $\alpha$-E59K and ATTO 532-labeled $\operatorname{ProT} \alpha$. Measuring the two species in parallel we collected $N \simeq 60$ hops on $\sim 5$ molecules and determine the charge of each species. We find an average difference in effective charge of $\Delta q_{\mathrm{m}}=2.6 \pm 1.5 e$ between the two species, which is in reasonable agreement with the theoretically expected value of $\Delta q_{\mathrm{c}}=1.4 e$ [Fig. 5].

\section{CONCLUSIONS}

We have demonstrated the ability to perform spectrally referenced high-precision measurements of the effective charge of single biological macromolecules in solution. Spectral calibration of the electrometry measurement is a key development that enables facile, direct measurements that are both accurate and precise.

Although the parameters that quantitatively influence the experiment are considered well known by direct measurement, the residual uncertainty in a key quantity, such as the slit height, has a non-negligible effect on the measurement accuracy. For example, spatial variations of the slit height of the order of about $1 \mathrm{~nm}$, which can occur over a distance of $\sim 50 \mu \mathrm{m}$, imply that unless the local height in the vicinity of a single molecule is accurately known, a single direct measurement under our current experimental conditions could carry an inaccuracy of $20 \%-30 \%$. The inaccuracy would increase for experiments performed at higher salt concentrations where the ratio $\frac{h_{\mathrm{e}}}{\kappa^{-1}}$ is even larger. While white light interferometry is capable of delivering direct measurements of gap heights and can even achieve sub-Angstrom precision on this measure, the accuracy is not expected to be better that about $1 \mathrm{~nm}$. Thus we use a molecule of known charge and size, and spectral characteristics distinct from the molecule of interest, in order to calibrate the entire measurement. This eliminates the need for averaging over multiple realizations in order to obtain an accurate result ${ }^{24}$ and could also potentially entirely obviate more demanding forms of setup characterization.

Future experiments will seek to simultaneously measure a large number of single molecules in parallel in order to characterize the spectrum of stable conformational or charged states populated by an ensemble of putatively identical macromolecules in solution. Since the current time resolution of the charge measurement is around $100 \mathrm{~ms}$, the approach will accurately probe molecular conformations that are stable over this observation timeframe. Tracking a molecule's transport in the lattice for a long enough total observation time will also permit real time monitoring of slow structural changes that occur on time scales of around $100 \mathrm{~ms}$ or longer.

\section{SUPPLEMENTARY MATERIAL}

See supplementary material for an example of spectrally resolved single-molecule electrometry measurement of 60bpATTO488 and 50bp-ATTO532.

\section{ACKNOWLEDGMENTS}

We gratefully acknowledge the support of the Swiss National Science Foundation, European Research Council, and the University of Zürich. We thank Franziska Zosel and Ben Schuler for the generous gift of fluorescently labeled $\operatorname{ProT} \alpha$. Nanofabrication was carried out at the FIRST Center for Micro- and Nanoscience, ETH Zürich.

${ }^{1}$ M. F. Perutz, "Electrostatic effects in proteins," Science 201, 1187-1191 (1978).

${ }^{2}$ A. Wada and H. Nakamura, "Nature of the charge distribution in proteins," Nature 293, 757-758 (1981).

${ }^{3}$ D. B. Thompson, J. J. Cronican, and D. R. Liu, "Engineering and identifying supercharged proteins for macromolecule delivery into mammalian cells," Methods Enzymol. 503, 293-319 (2012).

${ }^{4}$ M. S. Lawrence, K. J. Phillips, and D. R. Liu, "Supercharging proteins can impart unusual resilience," J. Am. Chem. Soc. 129, 10110-10112 (2007).

${ }^{5} \mathrm{~S}$. Mirceta, "Evolution of mammalian diving capacity traced by myoglobin net surface charge," Science 340, 1234192 (2013).

${ }^{6}$ S. C. L. Kamerlin, P. K. Sharma, R. B. Prasad, and A. Warshel, "Why nature really chose phosphate," Q. Rev. Biophys. 46, 1-132 (2013).

${ }^{7}$ A. Bah, R. M. Vernon, Z. Siddiqui, M. Krzeminski, R. Muhandiram, C. Zhao, N. Sonenberg, L. E. Kay, and J. D. Forman-Kay, "Folding of an intrinsically disordered protein by phosphorylation as a regulatory switch," Nature 519, 106-109 (2015).

${ }^{8}$ A. M. Bode and Z. G. Dong, "Post-translational modification of p53 in tumorigenesis," Nat. Rev. Cancer 4, 793-805 (2004).

${ }^{9}$ L. Martin, X. Latypova, and F. Terro, "Post-translational modifications of tau protein: Implications for Alzheimer's disease,” Neurochem. Int. 58, 458-471 (2011).

${ }^{10}$ J. T. G. Overbeek and M. J. Voorn, "Phase separation in polyelectrolyte solutions. Theory of complex coacervation,” J. Cell. Comp. Physiol. 49, 7-26 (1957).

${ }^{11}$ W. M. Aumiller and C. D. Keating, "Phosphorylation-mediated RNA/peptide complex coacervation as a model for intracellular liquid organelles," Nat. Chem. 8, 129-137 (2016).

${ }^{12}$ G. S. Manning, "Limiting laws and counterion condensation in polyelectrolyte solutions. I. colligative properties," J. Chem. Phys. 51, 924-933 (1969).

${ }^{13}$ G. S. Manning, "Molecular theory of polyelectrolyte solutions with applications to electrostatic properties of polynucleotides," Q. Rev. Biophys. 11, 179-246 (1978).

${ }^{14}$ G. S. Manning, "Electrostatic free energies of spheres, cylinders, and planes in counterion condensation theory with some applications," Macromolecules 40, 8071-8081 (2007).

${ }^{15}$ R. R. Netz and H. Orland, "Variational charge renormalization in charged systems," Eur. Phys. J. E 11, 301-311 (2003).

${ }^{16}$ B. W. Ninham and V. A. Parsegian, "Electrostatic potential between surfaces bearing ionizable groups in ionic equilibrium with physiologic saline solution," J. Theor. Biol. 31, 405-428 (1971).

${ }^{17}$ S. Alexander, P. M. Chaikin, P. Grant, G. J. Morales, P. Pincus, and D. Hone, "Charge renormalization, osmotic-pressure, and bulk modulus of colloidal crystals: Theory," J. Chem. Phys. 80, 5776-5781 (1984).

${ }^{18}$ M. Aubouy, E. Trizac, and L. Bocquet, "Effective charge versus bare charge: An analytical estimate for colloids in the infinite dilution limit," J. Phys. A: Math. Gen. 36, 5835-5840 (2003). 
${ }^{19}$ M. Lund and B. Jönsson, "Charge regulation in biomolecular solution," Q. Rev. Biophys. 46, 265-281 (2013).

${ }^{20} \mathrm{R}$. W. O'Brien and L. R. White, "Electrophoretic mobility of a spherical colloidal particle," J. Chem. Soc., Faraday Trans. 2 74, 1607-1626 (1978).

${ }^{21}$ J. M. Gao, M. Mammen, and G. M. Whitesides, "Evaluating electrostatic contributions to binding with the use of protein charge ladders," Science 272, 535-537 (1996).

${ }^{22}$ I. Gitlin, J. D. Carbeck, and G. M. Whitesides, "Why are proteins charged? Networks of charge-charge interactions in proteins measured by charge ladders and capillary electrophoresis," Angew. Chem., Int. Ed. 45, 3022-3060 (2006).

${ }^{23}$ J. D. Carbeck, J. C. Severs, J. Gao, Q. Wu, R. D. Smith, and G. M. Whitesides, "Correlation between the charge of proteins in solution and in the gas phase investigated by protein charge ladders, capillary electrophoresis, and electrospray ionization mass spectrometry," J. Phys. Chem. B 102, 10596-10601 (1998).

${ }^{24}$ F. Ruggeri, F. Zosel, N. Mutter, M. Różycka, M. Wojtas, A. Ożyhar, B. Schuler, and M. Krishnan, "Single-molecule electrometry," Nat. Nanotechnol. 12, 488-495 (2017).

${ }^{25}$ M. Krishnan, N. Mojarad, P. Kukura, and V. Sandoghdar, "Geometryinduced electrostatic trapping of nanometric objects in a fluid," Nature 467, 692-695 (2010).

${ }^{26}$ N. Mojarad and M. Krishnan, "Measuring the size and charge of single nanoscale objects in solution using an electrostatic fluidic trap," Nat. Nanotechnol. 7, 448-452 (2012).

${ }^{27} \mathrm{H}$. Kramers, "Brownian motion in a field of force and the diffusion model of chemical reactions," Physica 7, 284-304 (1940).

${ }^{28}$ M. Krishnan, "Electrostatic free energy for a confined nanoscale object in a fluid," J. Chem. Phys. 138, 114906 (2013).
${ }^{29}$ M. Krishnan, "A simple model for electrical charge in globular macromolecules and linear polyelectrolytes in solution,” J. Chem. Phys. 146, 205101 (2017).

${ }^{30}$ K. Gast, H. Damaschun, K. Eckert, K. Schulzeforster, H. R. Maurer, M. Mullerfrohne, D. Zirwer, J. Czarnecki, and G. Damaschun, "Prothymosin-alpha: A biologically-active protein with random coil conformation," Biochemistry 34, 13211-13218 (1995).

${ }^{31}$ S. Müller-Späth, A. Soranno, V. Hirschfeld, H. Hofmann, S. Rüegger, L. Reymond, D. Nettels, and B. Schuler, "Charge interactions can dominate the dimensions of intrinsically disordered proteins," Proc. Natl. Acad. Sci. U. S. A. 107, 14609-14614 (2010).

${ }^{32}$ M. Davies, B. Rühle, C. Li, K. Müllen, T. Bein, and C. Bräuchle, "Insights into nanoscale electrophoresis of single dye molecules in highly oriented mesoporous silica channels," J. Phys. Chem. C 118, 24013-24024 (2014).

${ }^{33}$ T. Kiuchi, M. Higuchi, A. Takamura, M. Maruoka, and N. Watanabe, "Multitarget super-resolution microscopy with high-density labeling by exchangeable probes," Nat. Methods 12, 743-746 (2015).

${ }^{34}$ B. V. Derjaguin, Theory of Stability of Colloids and Thin Films (Springer US, 1991).

${ }^{35}$ R. Sjoback, J. Nygren, and M. Kubista, "Characterization of fluoresceinoligonucleotide conjugates and measurement of local electrostatic potential," Biopolymers 46, 445-453 (1998).

${ }^{36} \mathrm{~K}$. Friederich and P. Woolley, "Electrostatic potential of macromolecules measured by $\mathrm{p} K_{\mathrm{a}}$ shift of a fluorophore. 1 . The $3^{\prime}$ terminus of $16 \mathrm{~s}$ RNA," Eur. J. Biochem. 173, 227-231 (1988).

${ }^{37}$ K. Friederich, P. Woolley, and K. G. Steinhäuser, "Electrostatic potential of macromolecules measured by $\mathrm{p} K_{\mathrm{a}}$ shift of a fluorophore. 2. Transfer RNA," Eur. J. Biochem. 173, 233-239 (1988). 